\title{
Picosecond dynamics of laser-induced strain in graphite
}

\author{
M. Harb, ${ }^{1}$ A. Jurgilaitis, ${ }^{1}$ H. Enquist, ${ }^{2}$ R. Nüske, ${ }^{1}$ C. v. Korff Schmising,,${ }^{1}$ J. Gaudin, ${ }^{3}$ S. L. Johnson, ${ }^{4}$ C. J. Milne,${ }^{5}$ P. Beaud, ${ }^{4}$ \\ E. Vorobeva, ${ }^{4}$ A. Caviezel, ${ }^{4}$ S. O. Mariager, ${ }^{4}$ G. Ingold, ${ }^{4}$ and J. Larsson ${ }^{1, *}$ \\ ${ }^{1}$ Atomic Physics Division, Department of Physics, Lund University, P.O. Box 118, 22100 Lund, Sweden \\ ${ }^{2}$ MAX-lab, Lund University, P.O. Box 118, Lund, Sweden \\ ${ }^{3}$ European XFEL GmbH, Albert-Einstein-Ring 19, D-22761 Hamburg, Germany \\ ${ }^{4}$ Swiss Light Source, Paul Scherrer Institut, 5232 Villigen PSI, Switzerland \\ ${ }^{5}$ Laboratorie de Spectroscopie Ultrarapide, Ecole Polytechnique Fèdèrale de Lausanne, 1015 Lausanne, Switzerland
}

(Received 5 May 2011; published 18 July 2011)

\begin{abstract}
We report on the use of grazing-incidence time-resolved $x$-ray diffraction to investigate the evolution of strain in natural graphite excited by femtosecond-laser pulses in the fluence range of $6-35 \mathrm{~mJ} / \mathrm{cm}^{2}$. Strains corresponding to up to $\sim 2.8 \% \mathrm{c}$-axis expansion were observed. We show that the experimental data is in good agreement with calculations based on the Thomsen strain model in conjunction with dynamical diffraction theory. Furthermore we find no evidence of nonthermal lattice expansion as reported in recent ultrafast electron-diffraction studies of laser-excited graphite conducted under comparable excitation conditions.
\end{abstract}

DOI: 10.1103/PhysRevB.84.045435

PACS number(s): 78.47.J-, 63.20.dd, 61.05.cp

\section{INTRODUCTION}

Ever since the discovery of fullerenes there has been an explosive interest in the synthesis of carbon-based materials, placing these materials at the forefront of the nanoscience revolution. Today carbon-based materials are the basis for numerous novel technologies including graphene based nanoelectronics ${ }^{1}$ and nanofluidics in carbon nanotubes. ${ }^{2}$ At the most basic level the existence of different carbon allotropes with rich and often exotic-material properties owes to the variety of bonding types that a carbon atom can form. Graphite is characterized by the weak van der Waals bonding between the interlayer planes and the strong covalent $\mathrm{sp}^{2}$-hybridized bonds in-plane. This bonding structure is responsible for the high anisotropy in the electronic, optical, and mechanical properties of graphite. The ability to modify the bonding configuration in graphite through irradiation by a laser pulse has been exploited to synthesize novel carbon structures such as nanodiamonds ${ }^{3,4}$ and $\mathrm{sp}^{3}$-rich carbon nanofoams. ${ }^{5}$ The ablation of intact layers of graphene off graphite has been also predicted $^{6}$ and demonstrated. ${ }^{7}$ While a detailed understanding of the processes leading to the change in the bonding configuration in laser-excited graphite is still lacking, it is believed that three key mechanisms are involved: The abrupt change in the electronic configuration because of the excitation of the $\pi$ electrons, the ensuing launch of large-amplitude coherent optical-phonons, and the thermal strains that develop at relatively later times. The strain-wave dynamics studied in this paper are sensitive to the interlayer-binding strength and should lead to an improved understanding of thermal stress and strain effects in graphite. Given the important role of strain in modulating the electronic properties of graphene, ${ }^{8}$ understating of stress and strain effects is essential to the successful application of graphene in the next generation of electronics. To further our understanding of strain-wave dynamics and other laser-induced electron-phonon processes, one would like to gain a glimpse of the dynamics that follow optical excitation from an atomic perspective and on the relevant femtosecond to picosecond timescales. Capturing transient atomic structures with such unprecedented time resolution became recently possible because of advances in time-resolved x-ray and electron-diffraction techniques. ${ }^{9-16}$ These techniques, with the ability to directly probe the structure of the perturbed lattice, offer extraordinary insights into the complex and competing channels of relaxation that follow the interaction of laser with matter.

In a recent study by Carbone et al., laser-excited graphite was investigated using ultrafast electron diffraction (UED) in reflection geometry. ${ }^{15}$ Carbone and coworkers observed contraction of the graphite lattice along the $c$ axis at the onset of excitation, followed by expansion of up to $1.25 \%$ of the interlayer distance at the highest fluence of $44.5 \mathrm{~mJ} / \mathrm{cm}^{2}$. It was argued that such a large amount of lattice expansion cannot be accounted for by linear-thermal expansion alone. Instead, the authors attributed the expansion to nonthermal mechanisms that include the anisotropic population of carriers in the electronic band and the subsequent generation of coherent optical-phonons. The results reported by Carbone et al. started a debate within the scientific community on whether the observed shifts in the positions of the diffraction spots represent real structural dynamics or are somehow related to transient electric fields generated at the surface of the sample because of the ejection of electrons by the laser pulse. Park et al. investigated the effects of transient-electric fields on an electron-probe pulse both experimentally and through simulations and concluded that these fields can indeed deflect the electron probe in a way consistent with the dynamics observed in Carbone's work. ${ }^{17,18}$ The claims of Park et al., however, were recently disputed. ${ }^{19,20}$ In yet another graphite study by Raman et al., performed under similar excitation conditions and using the same technique of UED, even larger amounts of shifts in the positions of the diffraction spots were reported, corresponding to $6 \%$ expansion of the interlayer distance at an excitation fluence of $40 \mathrm{~mJ} / \mathrm{cm}^{2} .{ }^{16}$ Raman et al. attributed the shifts partially to structural dynamics and partially to surface charging of the sample and presented a model of the surface potential, which was used to separate the two effects. It is evident from the forgoing survey of conflicting results that in order to settle the question of whether or not laser excitation of graphite gives rise to nonthermal strains, an alternative 
approach to UED is warranted. To this end, here we investigate the structural dynamics of laser-irradiated graphite using time-resolved $x$-ray diffraction. $X$ rays make an ideal structural probe in this context as they are insensitive to transientelectric fields that may be generated at the surface of the sample.

\section{EXPERIMENTAL SETUP}

The experiment was carried out at the Swiss Light Source (SLS). The FEMTO slicing-source at SLS generates 140-fs $\mathrm{X}$-ray pulses through laser slicing of the electrons in the insertion device. The overall time resolution of the pump-probe scheme is dictated by the duration of the $\mathrm{x}$-ray probe and laser-pump pulses, the timing jitter of the laser system, and the geometrical mismatch angle between pump and probe beams. An overall time resolution of $<200$ fs has been demonstrated in several recent studies performed at this beamline (e.g., see Johnson et $a{ }^{21}{ }^{21}$ ). The sample used in our study is a mined natural graphite flake, which was cleaved to produce a surface with high-quality flatness. The single-crystalline nature of the sample was verified through static x-ray diffraction measurements carried out at beamline D611 at MAX-lab. For the time-resolved measurements, the $\mathrm{x}$-ray source was tuned to an energy of $5.85 \mathrm{KeV}$ with $\sim 1 \%$ bandwidth and focused to a spot size of $\sim 300 \times 10 \mu \mathrm{m}^{2}$. To match the $\mathrm{x}$-ray penetration depth with the optical-absorption depth in graphite, we employ the noncoplanar-diffraction geometry with extreme (near critical angle) grazing incidence shown in Fig. 1(a). The grazing angle $\left(\varphi_{\mathrm{i}}\right)$ was set by first calculating the corresponding vertical displacement of the specularly reflected x-ray beam relative to the direct beam on a screen positioned at known distance from the sample. An avalanche photodiode (APD) was then placed at the set displacement and the intensity of the speculary reflected $\mathrm{x}$-ray beam was maximized by varying $\varphi_{\mathrm{i}}$. Measurements were conducted at two different grazing angles below $\left(0.27^{\circ}\right)$ and above $\left(0.50^{\circ}\right)$ the critical angle of $0.30^{\circ}$. The sample was excited at fluences ranging from $6-35 \mathrm{~mJ} / \mathrm{cm}^{2}$ with $p$-polarized 120 -fs-laser pulses centered around $800 \mathrm{~nm}$ and incident at $10^{\circ}$ relative to the sample surface. The $0.75 \times 4.5 \mathrm{~mm}^{2}$ footprint of the laser beam on the sample was sufficiently large to cover the $0.3 \times 2 \mathrm{~mm}^{2} \mathrm{x}$-ray footprint, ensuring homogeneous excitation of the probed area. $\mathrm{X}$-ray rocking curves were recorded at selected time delays in the -10 ps to +100 ps range by measuring the intensity of the 101 reflection with an APD as function of the sample in plane rotation $\left(\theta_{\mathrm{i}}\right)$. The respective repetition rates of the $\mathrm{x}$-ray probe and laser-pump pulses of $2 \mathrm{KHz}$ and $1 \mathrm{KHz}$ imply that every other X-ray pulse contributes to a reference (unpumped) signal. Figure 1(b) shows the 101 rocking curve of the unpumped sample at a grazing angle of $0.27^{\circ}$.

\section{RESULTS AND THEORETICAL MODEL}

Results of time-resolved measurements are shown in Fig. 2 in the form of rocking-curve differences at selected time points both below (a-f) and above $(\mathrm{g}-\mathrm{l})$ critical angle and for an incident excitation fluence of $26 \mathrm{~mJ} / \mathrm{cm}^{2}$. It is evident from the time-dependent shift of the rocking curves that the graphite lattice is strained. To explain the detailed shape of the curves,
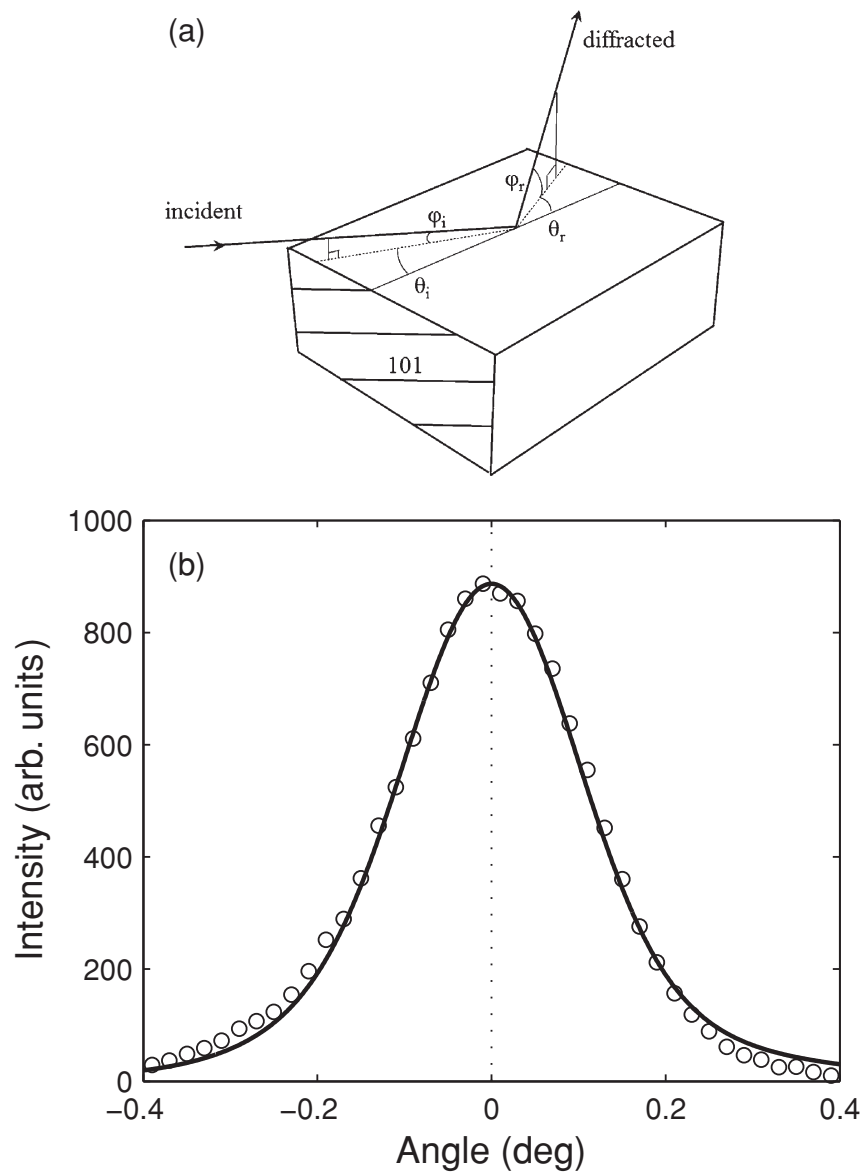

FIG. 1. (a) The noncoplanar-diffraction geometry of the experiment. $5.85 \mathrm{KeV} x$-rays incident at a grazing angle of $<0.5^{\circ}$ relative to the surface of the crystal diffract off the 101 lattice planes. The diffracted beam is deflected by $\sim 62^{\circ}$ azimuthally $\left(\theta_{\mathrm{i}}+\theta_{\mathrm{r}}\right)$ and by $\sim 18^{\circ}$ relative to the surface of the crystal $\left(\varphi_{\mathrm{r}}\right)$. (b) Measured (open circles) and calculated (solid line) rocking curve of the 101 reflection.

modeling of strain-wave propagation and x-ray scattering is needed. We employ the strain model proposed by Thomsen et $a l .{ }^{22}$ in which the laser-excited sample is assumed to develop instantaneous thermal stress of the form $-3 B \beta \Delta T(z)$, where $B$ is the bulk modulus, $\beta$ is the linear expansion coefficient, and $\Delta T(z)$ is the temperature profile along the sample depth $(z)$. The finite coupling time between electrons and lattice can be accounted for by considering the following time dependent temperature profile

$$
\Delta T(z, t)=\frac{(1-R) F}{C \delta}[1-\exp (-t / \tau)] \exp (-z / \delta),
$$

where $R$ is the reflectivity, $F$ is the incident fluence, $C$ is the volumetric heat capacity, $\delta$ is the optical absorption depth, and $\tau$ is the electron-phonon coupling time constant. When the finite coupling time is introduced, the analytical expression for the strain propagation given by Thomsen et al. can no longer be used. Instead the strain propagation is calculated numerically. The numerical solution of the relevant equations of elasticity is presented in Fig. 3(a) in the form of a spatio-temporal map of the $c$-axis strain. Note the maximum strain of $\sim 2.5 \%$ at the surface corresponding to a $\sim 1000 \mathrm{~K}$ temperature change and a 

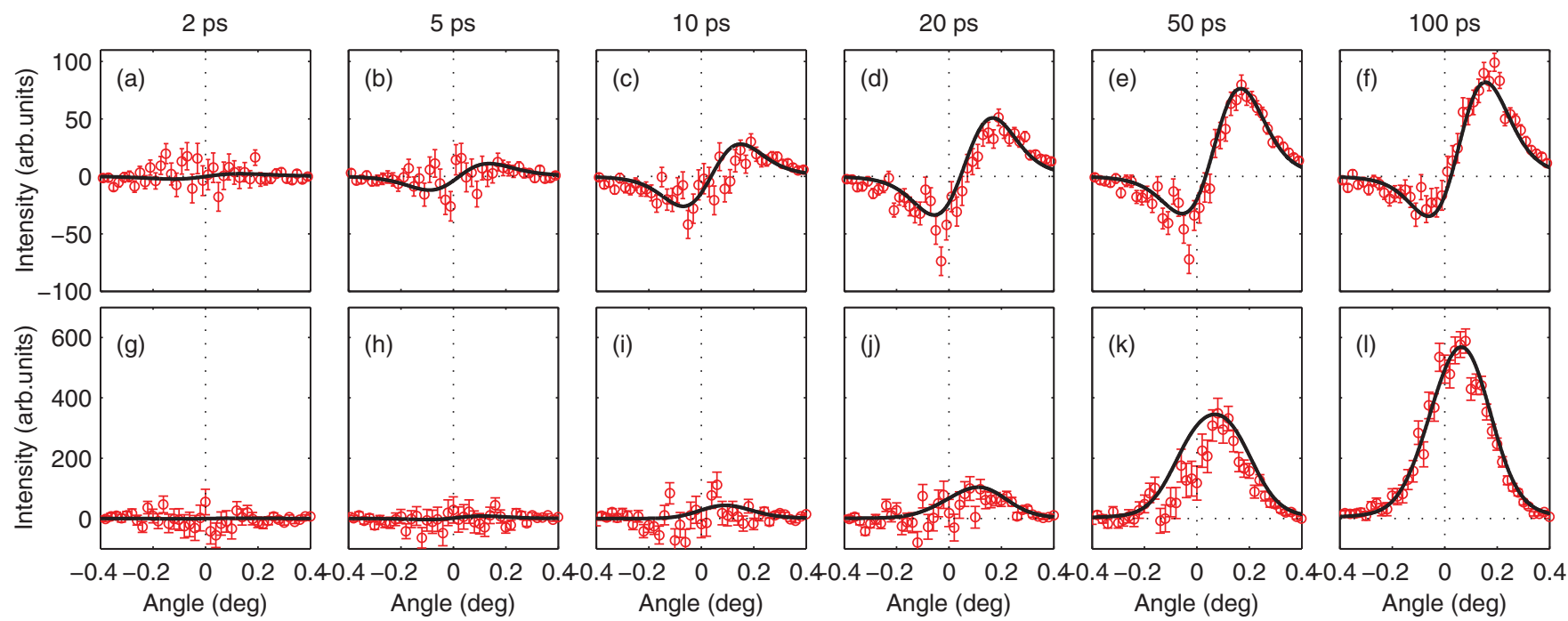

FIG. 2. (Color online) Rocking-curve differences taken at selected time delays relative to laser excitation at $26 \mathrm{~mJ} / \mathrm{cm}^{2}$. Two sets of measurements were taken: (a-f) grazing angle of $0.27^{\circ}$ (below critical angle); (g-1) grazing angle of $0.5^{\circ}$ (above critical angle). Open circles with error bars are experimental data points and solid lines are dynamical-diffraction calculations of a thermally strained crystal.

$c$-axis thermal-expansion coefficient of $27 \times 10^{-6} \mathrm{~K}^{-1} \cdot{ }^{23} \mathrm{An}$ electron-phonon coupling constant of 8 ps was assumed. ${ }^{15,16}$ In addition we verified by solving the heat-diffusion equation that the effects of heat diffusion can be neglected because of the relatively low thermal conductivity along the $c$ axis of graphite. ${ }^{24}$ This assumption is also supported by Carbone's study, in which following the initial drop in the diffracted intensity, no significant changes are observed up to $\sim 1 \mathrm{~ns} .{ }^{15}$

The spatio-temporal map of strain was used to create a deformed lattice structure of graphite and subsequently calculate the x-ray diffraction intensity of the deformed structure using dynamical diffraction theory performed on the
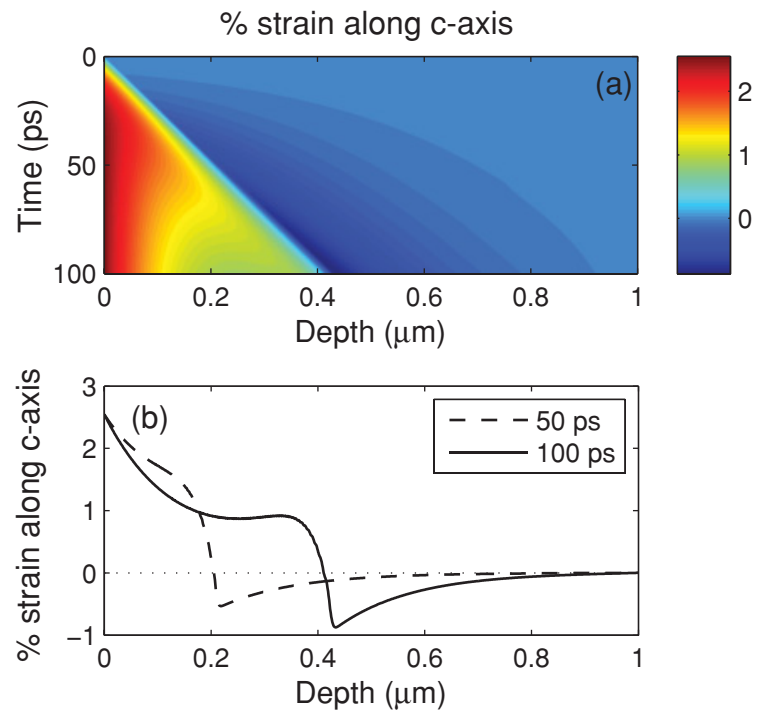

FIG. 3. (Color online) (a) Spatio-temporal map of the $\%$ strain along $c$ axis of graphite excited at $26 \mathrm{~mJ} / \mathrm{cm}^{2}$. The strain was numerically calculated according to the Thomsen model. ${ }^{22}$ (b) Strain profile at $+50 \mathrm{ps}$ (dashed) and $+100 \mathrm{ps}$ (solid).
Stepanov X-ray Server. ${ }^{25}$ The $\sim 0.05^{\circ}$ divergence of the $\mathrm{x}$-ray beam was accounted for by performing simulations at different grazing angles and weight averaging the results according to a Gaussian distribution of angles. The $1.2 \%$ bandwidth of the x-ray beam was also accounted for by convoluting the weight-averaged result with a voigt lineshape. The calculated rocking curve for zero strain (unpumped sample) is shown as solid line in Fig. 1(b). Calculated rocking-curve differences at selected time points are shown as solid lines in Fig. 2(a-1). It is evident from the good agreement between measurements and calculations that the Thomsen model provides an accurate description of strain dynamics. Within our model we found the shape of the simulated rocking-curve differences to be sensitive to the optical absorption depth $\delta$ beyond a simple scaling factor. Therefore, we treated $\delta$ as a free-fit parameter and found the value of $\sim 140 \mathrm{~nm}$ to best reproduce the measured rocking-curve differences. Note that this value of $\delta$ is within what has been observed experimentally. ${ }^{4}$

\section{DISCUSSION}

Qualitatively, the time-dependent features of the diffraction profiles can be understood as follows. Below critical angle, the $\mathrm{x}$-ray absorption depth is less than the optical-absorption depth of $\sim 140 \mathrm{~nm}^{4}$ Strain is initially confined to the surface but evolves over time to mimic the laser-absorption profile. As strain waves propagate deeper into the material, more atomic layers become disturbed, but the average strain within the disturbed region is reduced. This is clearly seen by comparing the strain profiles at $50 \mathrm{ps}$ and $100 \mathrm{ps}$ in Fig. 3(b). With respect to $\mathrm{x}$-ray diffraction, this simple picture explains both the increase in the intensity of the rocking-curve difference with time and the monotonic shift toward smaller angles of the zero crossing (intersection of rocking-curve difference and $x$ axis) indicating reduced strain. We note that at these shallow angles, strain estimated directly from the raw data as double the value of the zero crossing, ${ }^{26}$ agrees well with calculated 


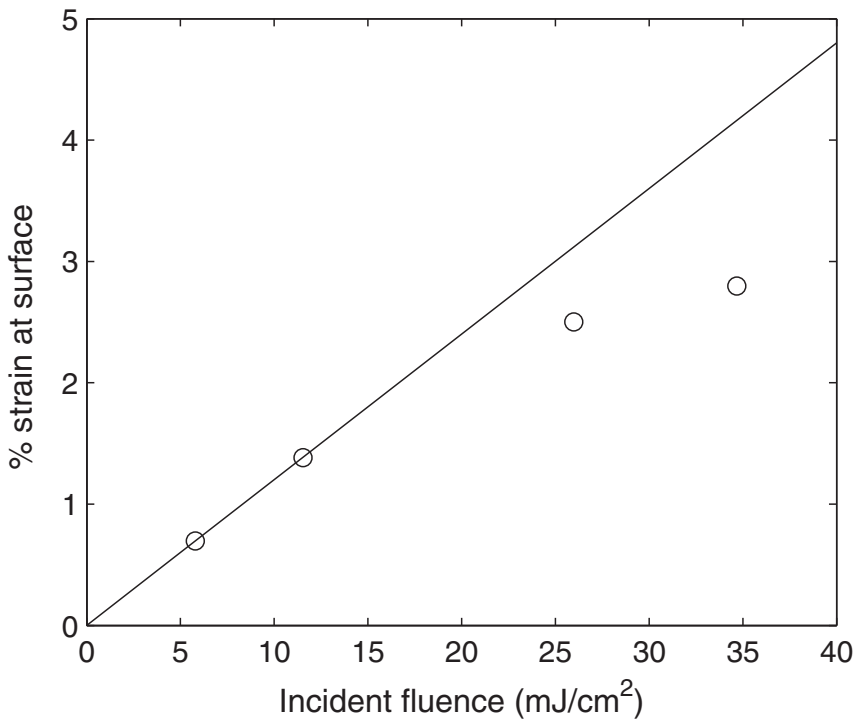

FIG. 4. Measured strain at $+50 \mathrm{ps}$ for experiments conducted at different excitation fluences.

strain. The maximum amount of shift of the 101 rocking curve of $0.16^{\circ}$, observed at $+10 \mathrm{ps}$, corresponds to a $c$-axis expansion of $2.4 \%$. Another interesting feature of the dynamics is the asymmetric character of the rocking-curve differences. This complex feature originates from the portion of the x-ray beam that by virtue of its large divergence penetrates deeper into the sample. Above critical angle the $\mathrm{x}$-ray absorption depth is much larger than the optical-absorption depth. The rocking-curve difference for these measurements loses the character of a simple shift of a diffraction peak with conserved amplitude and width. For these measurements, strain cannot be directly extracted from the raw data. Nevertheless, employing dynamical-diffraction theory faithfully reproduces the observed features, as evident in Fig. 2(g-1).

A summary of the fluence-dependent measurements is shown in Fig. 4 in the form of surface strain at +50 ps as function of incident fluence. Note that the deviation from linearity is similar in character to the saturation of the atomic mean-square displacements above $\sim 20 \mathrm{~mJ} / \mathrm{cm}^{2}$ observed by Raman et al. ${ }^{15}$ We verified that the nonlinearily cannot be attributed to a change in the optical reflectivity of the sample since the measured reflectivity was found to be constant up to the damage threshold of $\sim 100 \mathrm{~mJ} / \mathrm{cm}^{2}$. However, we cannot rule out the possibility that the saturation effect is related to an increase in the optical-absorption depth with fluence. Evidence for such effect is implied from recent optical pump-probe measurements, in which laser excitation induced a transient increase in the transmittivity of graphene and graphite films. ${ }^{27,28}$

We discuss our results in light of the recent UED studies of graphite. ${ }^{15,16}$ Carbone et al. came to the conclusion of nonthermal strains based on an estimate of the temperature change of the sample of $40 \mathrm{~K}$ at $44.5 \mathrm{~mJ} / \mathrm{cm}^{2}$ and a thermal expansion coefficient of $7.9 \times 10^{-6} \mathrm{~K}^{-1}$. First, we note that the relevant thermal-expansion coefficient in all of these experiments is not the $7.9 \times 10^{-6} \mathrm{~K}^{-1}$ of isotropic graphite but the $27 \times$ $10^{-6} \mathrm{~K}^{-1}$ along the $c$ axis of natural graphite. ${ }^{23}$ Second we believe Carbone's estimate of temperature change to be significantly underestimated. Our estimate of temperature change of $\sim 1000 \mathrm{~K}$ at $26 \mathrm{~mJ} / \mathrm{cm}^{2}$ and Raman et al.'s estimate of $950 \mathrm{~K}$ at $21 \mathrm{~mJ} / \mathrm{cm}^{2}$ suggest a temperature change of around $2000 \mathrm{~K}$ at $44.5 \mathrm{~mJ} / \mathrm{cm}^{2}$. We note here that a $2000 \mathrm{~K}$ temperature change can explain the $\sim 50 \%$ drop in the intensity of the 0014 reflection in Carbone's study in accordance with the Debye-Waller effect. Furthermore Carbone et al. argues that the observed nonthermal strains are related to the excitation of the so-called strongly coupled optical phonons (SCOP). ${ }^{29,30}$ However, the measured lifetime of SCOP, 5-7 ps, ${ }^{29,30}$ does not support the persistence of nonthermal strains up to $\sim 1 \mathrm{~ns}$, as observed in Carbone's work. Based on the previous accounts, we believe that the $\sim 1.25 \%$ positive strain in Carbone's study is purely thermal in nature. Another interesting feature of the UED studies is the detection of negative strains within picoseconds following excitation. The amount of $c$-axis contraction varies from $\sim 0.03 \%$ in Carbone's study to $\sim 5 \%$ in Raman's study. In our data we see no clear evidence of a negative strain component. We have carried out additional simulations to set a limit on the lowest negative strain we can observe. Since the negative stress is short lived, it gives rise to strain confined to the topmost $\sim 5 \mathrm{~nm}$ of the sample. Given our surface sensitivity and the signal-to-noise ratio this negative strain component cannot be larger than $0.5 \%$ for the $26 \mathrm{~mJ} / \mathrm{cm}^{2}$ measurements.

\section{CONCLUSION}

In conclusion our experiment supports the measurement of positive strains by Carbone et al. enforcing that these strains represent real structural dynamics. However, we disagree with the interpretation of the data suggesting that the observed strains point to a nonthermal contribution to the expansion of the lattice. To the contrary we believe that the saturation effect in the atomic displacements in our experiment and in Raman's study indicate that the measured positive strains above $20 \mathrm{~mJ} / \mathrm{cm}^{2}$ are smaller than what is expected from thermal expansion. Finally this work demonstrates timeresolved $\mathrm{x}$-ray diffraction in grazing geometry as a tool for resolving structural changes in light elements that do not efficiently scatter $x$ rays. The new generation of light sources, with their superior beam qualities, will have sufficient surface sensitivity to fully explore the contraction effect that is thought to be limited to the topmost few layers in graphite.

\section{ACKNOWLEDGMENTS}

These experiments were performed at the FEMTO source at the X05LA beamline at the Swiss Light Source, Paul Scherrer Institut, Villigen, Switzerland. The authors thank the Swedish Research Council (VR), the Knut and Alice Wallenberg Foundation, the Crafoord Foundation, and the Carl Trygger Foundation for financial support. M.H. acknowledges financial support from the Natural Sciences and Engineering Research Council of Canada. This research project has been supported by the European Commission under the 7th Framework Programme: Research Infrastructures. Grant Agreement Number 226716. 
*jorgen.larsson@fysik.lth.se

${ }^{1}$ M. Freitag, Nature Nanotechnology 3, 455 (2008).

${ }^{2}$ A. Noy, H. G. Park, F. Fornasiero, J. K. Holt, C. P. Grigoropoulos, and O. Bakajin, Nano Today 2, 22 (2007).

${ }^{3}$ G. W. Yang and J. B. Wang, Appl. Phys. A 72, 475 (2001).

${ }^{4}$ A. Hu, M. Rybachuk, Q.-B. Lu, and W. W. Duley, Appl. Phys. Lett. 91, 131906 (2007).

${ }^{5}$ A. V. Rode, E. G. Gamaly, and B. Luther-Davies, Appl. Phys. A 70, 135 (2000).

${ }^{6}$ H. O. Jeschke, M. E. Garcia, and K. H. Bennemann, Phys. Rev. Lett. 87, 015003 (2001).

${ }^{7}$ A. Kaplan, M. Lenner, and R. E. Palmer, Phys. Rev. B 76, 073401 (2007).

${ }^{8}$ V. M. Pereira and A. H. Castro Neto, Phys. Rev. Lett. 103, 046801 (2009).

${ }^{9}$ A. Rousse, C. Rischel, S. Fourmaux, I. Uschmann, S. Sebban, G. Grillon, Ph. Balcou, E. Förster, J. P. Geindre, P. Audebert, J. C. Gauthier, and D. Hulin, Nature 410, 65 (2001).

${ }^{10}$ A. M. Lindenberg, J. Larsson, K. Sokolowski-Tinten, K. J. Gaffney, C. Blome, O. Synnergren, J. Sheppard, C. Caleman, A. G. MacPhee, D. Weinstein, D. P. Lowney, T. K. Allison, T. Matthews, R. W. Falcone, A. L. Cavalieri, D. M. Fritz, S. H. Lee, P. H. Bucksbaum, D. A. Reis, J. Rudati, P. H. Fuoss, C. C. Kao, D. P. Siddons, R. Pahl, J. Als-Nielsen, S. Duesterer, R. Ischebeck, H. Schlarb, H. Schulte-Schrepping, Th. Tschentscher, J. Schneider, D. von der Linde, O. Hignette, F. Sette, H. N. Chapman, R. W. Lee, T. N. Hansen, S. Techert, J. S. Wark, M. Bergh, G. Huldt, D. van der Spoe, N. Timneanu, J. Hajdu, R. A. Akre, E. Bong, P. Krejcik, J. Arthur, S. Brennan, K. Luening, and J. B. Hastings, Science 308, 392 (2005).

${ }^{11}$ J. Larsson, A. Allen, P. H. Bucksbaum, R. W. Falcone, A. Lindenberg, G. Naylor, T. Missalla, D. A. Reis, K. Scheidt, A. Sjögren, P. Sondhauss, M. Wulff, and J. S. Wark, Appl. Phys. A: Mater. Sci. Process. 75, 467 (2002).

${ }^{12}$ R. J. D. Miller, R. Ernstorfer, M. Harb, M. Gao, C. T. Hebeisen, H. Jean-Ruel, C. Lu, G. Moriena, and G. Sciaini, Acta Cryst. A 66, 137 (2010).

${ }^{13}$ M. Harb, R. Ernstorfer, C. T. Hebeisen, G. Sciaini, W. Peng, T. Dartigalongue, M. A. Eriksson, M. G. Lagally, S. G. Kruglik, and R. J. D. Miller, Phys. Rev. Lett. 100, 155504 (2008).
${ }^{14}$ J. Cao, Z. Hao, H. Park, C. Tao, D. Kau, and L. Blaszczyk, Appl. Phys. Lett. 83, 1044 (2003).

${ }^{15}$ F. Carbone, P. Baum, P. Rudolf, and A. H. Zewail, Phys. Rev. Lett. 100, 035501 (2008).

${ }^{16}$ R. K. Raman, Y. Murooka, C.-Y. Ruan, T. Yang, S. Berber, and D. Tománek, Phys. Rev. Lett. 101, 077401 (2008).

${ }^{17}$ H. Park and J. M. Zuo, Appl. Phys. Lett. 94, 251103 (2009).

${ }^{18}$ H. Park and J. M. Zuo, Phys. Rev. Lett. 105, 059603 (2010).

${ }^{19}$ F. Carbone, P. Baum, P. Rudolf, and A. H. Zewail, Phys. Rev. Lett. 105, 059604 (2010).

${ }^{20}$ S. Schäfera, W. Lianga, and A. H. Zewail, Chem. Phys. Lett. 493, 11 (2010).

${ }^{21}$ S. L. Johnson, P. Beaud, E. Vorobeva, C. J. Milne, É. D. Murray, S. Fahy, and G. Ingold, Phys. Rev. Lett. 102, 175503 (2009).

${ }^{22}$ C. Thomsen, H. T. Grahn, H. J. Maris, and J. Tauc, Phys. Rev. B 34, 4129 (1986).

${ }^{23}$ E. A. Kellett and B. P. Richards, J. Appl. Cryst. 4, 1 (1971).

${ }^{24}$ K. Sun, M. A. Stroscio, and M. Dutta, Superlattices Microstruct. 45, 60 (2009).

${ }^{25} \mathrm{~S}$. Stepanov, in Advances in Computational Methods for X-ray and Neutron Optics, edited by M. Sanches del Rio, Proceedings SPIE, 5536, pp. 16-26, (2004).

${ }^{26}$ Note that the distance between the maxima and minima of the rocking curve difference is an incorrect measure of the rocking curve shift. This can be easily verified by considering the difference between two Gaussian curves of equal amplitudes and widths shifted by an amount that is much less than the width.

${ }^{27}$ J. M. Dawlaty, S. Shivaraman, M. Chandrashekhar, F. Rana, and M. G. Spencer, Appl. Phys. Lett. 92, 042116 (2008).

${ }^{28}$ F. Carbone, G. Aubock, A. Cannizzo, F. Van Mourik, R. R. Nair, A. K. Geim, K. S. Novoselov, and M. Chergui, Chem. Phys. Lett. 504, 37 (2011).

${ }^{29}$ T. Kampfrath, L. Perfetti, F. Schapper, C. Frischkorn, and M. Wolf, Phys. Rev. Lett. 95, 187403 (2005).

${ }^{30}$ H. Yan, D. Song, K. F. Mak, I. Chatzakis, J. Maultzsch, and T. F. Heinz, Phys. Rev. B 80, 121403 (2009). 